\title{
EXISTENCE OF WEAK SOLUTIONS \\ FOR A SCALE SIMILARITY MODEL \\ OF THE MOTION OF LARGE EDDIES \\ IN TURBULENT FLOW
}

\section{MERYEM KAYA}

Received 28 January 2003 and in revised form 5 May 2003

In turbulent flow, the normal procedure has been seeking means $\bar{u}$ of the fluid velocity $u$ rather than the velocity itself. In large eddy simulation, we use an averaging operator which allows for the separation of largeand small-length scales in the flow field. The filtered field $\bar{u}$ denotes the eddies of size $O(\delta)$ and larger. Applying local spatial averaging operator with averaging radius $\delta$ to the Navier-Stokes equations gives a new system of equations governing the large scales. However, it has the wellknown problem of closure. One approach to the closure problem which arises from averaging the nonlinear term is the use of a scale similarity hypothesis. We consider one such scale similarity model. We prove the existence of weak solutions for the resulting system.

\section{Introduction}

The turbulent flow of an incompressible fluid is modelled by solution $(u, p)$ of the incompressible Navier-Stokes equations

$$
\begin{gathered}
u_{t}+\nabla \cdot(u u)-\operatorname{Re}^{-1} \Delta u+\nabla p=f \quad \text { in } \Omega, \text { for } 0<t \leq T, \\
\nabla \cdot u=0 \quad \text { in } \Omega, \text { for } 0<t \leq T, \\
u(x, 0)=u_{0}(x) \quad \text { in } \Omega, u=0 \text { on } \partial \Omega, \text { for } 0<t \leq T, \\
\int_{\Omega} p d x=0,
\end{gathered}
$$

where $\Omega \subset \mathbb{R}^{d}(d=2$ or 3$), u: \Omega \times[0, T] \rightarrow \mathbb{R}^{d}$ is the fluid velocity, $p: \Omega \rightarrow$ $\mathbb{R}$ is the fluid pressure, $f(x, t)$ is the (known) body force, $u_{0}(x)$ is the initial flow field, and Re is the Reynolds number. There are numerous 
approaches to the simulation of turbulent flows in practical settings. One of the most promising current approaches is large eddy simulation (LES) in which approximations to local spatial averages of $u$ are calculated. In LES, the filtered quantities and fluctuations are defined as

$$
\begin{gathered}
\bar{u}(x, t)=g_{\delta} * u=\int_{\mathbb{R}^{3}} g_{\delta}\left(x-x^{\prime}\right) u\left(x^{\prime}, t\right) d x^{\prime}, \\
u^{\prime}=u-\bar{u},
\end{gathered}
$$

where

$$
g_{\delta}=\delta^{-3} g\left(\frac{x}{\delta}\right)
$$

and $g$ is the filter function of characteristic width $\delta$. Applying the filtering operator to the Navier-Stokes equations gives

$$
\bar{u}_{t}+\nabla \cdot(\overline{u u})-\operatorname{Re}^{-1} \Delta \bar{u}+\nabla \bar{p}=\bar{f}, \quad \nabla \cdot \bar{u}=0, \text { in } \Omega \times(0, T] .
$$

The governing equation (1.4) may be rewritten as

$$
\bar{u}_{t}+\nabla \cdot(\bar{u} \bar{u})-\operatorname{Re}^{-1} \Delta \bar{u}+\nabla \bar{p}+\nabla \overline{\mathbb{T}}=\bar{f}, \quad \nabla \cdot \bar{u}=0, \text { in } \Omega \times(0, T],
$$

where $\mathbb{T}$ denotes the subgrid tensor defined as

$$
\mathbb{T}:=\bar{u} u-\bar{u} \bar{u}
$$

which must be modelled. In general, the approach to closure in LES, based on the scale similarity hypothesis, was introduced in 1980 by Bardina et al. [1]. The idea of scale similarity can be thought of as a sort of extrapolation from the resolved scales to the unresolved scales. The original Bardina model is given by

$$
\bar{u} \bar{u}-\bar{u} \bar{u} \cong \overline{\bar{u}} \bar{u}-\overline{\bar{u}} \overline{\bar{u}}
$$

This model has proved to be highly consistent [11, 12], but stability problems have been reported in various tests of the Bardina model. These have led to various extensions of Bardina model such as the Layton model proposed in [8], the Liu-Meneveau-Katz model [10], Horiuti's filtered Bardina model [4], and many "mixed" models. In this paper, we consider a model proposed in [8], which is another realization of the idea of scale similarity seeking a clear kinetic energy balance. The model is based on the following three modelling steps and the nonlinear term 
is written as [9]

$$
\overline{u u}=\overline{\bar{u}} \bar{u}+\overline{\bar{u} u^{\prime}+u^{\prime} \bar{u}}+\overline{u^{\prime} u^{\prime}} .
$$

Step 1 . The cross terms are modelled by scale similarity:

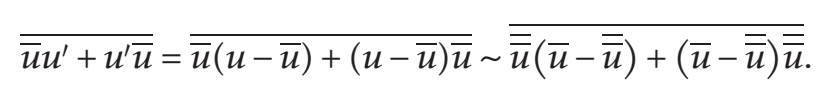

Step 2. The resolved term $\overline{\bar{u}} \bar{u}$ is modelled with a Boussinesq-type assumption

$$
\overline{\bar{u}} \bar{u} \sim \overline{\bar{u}} \overline{\bar{u}}+\text { dissipative mechanism on } O(\delta) \text { scales, }
$$

where

$$
\nabla \cdot(\overline{\bar{u}} \bar{u}) \sim \nabla \cdot(\overline{\bar{u}} \overline{\bar{u}})-A(\delta) \bar{u} .
$$

The operator $A(\delta) w$ takes the general form $A(\delta) w=R^{*} \nabla \cdot \mathbb{T}_{F}(R w)$, where $R$ is a restriction operator to the finest resolved scales. It is defined by the use of its variational representation

$$
-(A(\delta) w, v)=\left(v_{F}(\delta) \mathbb{D}(w-\bar{w}), \mathbb{D}(v-\bar{v})\right),
$$

where $v_{F}(\delta)$ is the fine scale fluctuation coefficient. This is simplified to

$$
A(\delta) w \sim \nabla \cdot\left(v_{F}(\delta) \mathbb{D}(w-\bar{w})-(w-\overline{\bar{w}})\right)
$$

where $\mathbb{D}(w):=(1 / 2)\left(\nabla w+\nabla w^{t}\right)$.

Step 3 . The $\overline{u^{\prime} u^{\prime}}$ term is modelled by a Boussinesq hypothesis that

$$
\overline{u^{\prime} u^{\prime}} \sim-v_{T}(\delta, \bar{u})\left(\nabla \bar{u}+\nabla \bar{u}^{t}\right),
$$

where $v_{T}(\delta, \bar{u})$ is called turbulent viscosity coefficient. Using (1.9), (1.11), and $(1.14)$ in $(1.4)$, the model is written with respect to $(w, q)$ which denotes the resulting approximation to $(\bar{u}, \bar{p})$,

$$
\begin{aligned}
& w_{t}+\nabla \cdot(\bar{w} \bar{w})+\nabla \cdot(\overline{\bar{w}}(w-\bar{w})+(w-\bar{w}) \bar{w})-\nabla \cdot\left(\mathcal{v}_{T}(\delta, w)\left(\nabla w+\nabla w^{t}\right)\right) \\
& -\nabla q-\operatorname{Re}^{-1} \Delta w-A(\delta) w=\bar{f}, \quad \nabla \cdot w=0, \text { in } \Omega \times(0, T],
\end{aligned}
$$


432 Existence of weak solutions for a scale similarity model

where $w, f: \Omega \times[0, T] \rightarrow \mathbb{R}^{d}, q: \Omega \rightarrow \mathbb{R}$. Boundary and zero mean conditions must be imposed on (1.15). There are several possibilities for the turbulent viscosity coefficient. The most common ones used in computational practice are a bulk viscosity $\mathcal{v}_{T}=\mathcal{v}_{T}(\delta)$, the viscosity of [5], $\boldsymbol{v}_{T}=$ $(0.17) \delta|w-\bar{w}|$, and the Smagorinsky model, see $[2,6,7,13]$,

$$
v_{T}(\delta, w)=\left(c_{S} \delta\right)^{2}\left|\nabla w+\nabla w^{t}\right|
$$

We will assume that $\boldsymbol{v}_{T}=0$, namely, there is no extra viscosity terms. With (1.16) or $v_{T}=v_{T}(\delta)$, our results can be easily extended. Before starting to prove the existence of weak solution for the model, we will give a proof that the model, given by (1.15), is Galilean invariant. It has been shown that the filtered form of Navier-Stokes equation is Galilean invariant [14]. Thus, it is enough to show that

$$
\nabla \cdot(\tilde{\mathbb{T}}(w+W))=\nabla \cdot \tilde{\mathbb{T}}(w)
$$

for any constant vector $W$. To this end we will give the following lemma.

LEMMA 1.1. Consider the model of the subgrid tensor

$$
\begin{aligned}
\mathbb{T}= & \overline{u u}-\bar{u} \bar{u} \sim \bar{w} \bar{w}+\overline{\bar{w}}(w-\bar{w})+(w-\bar{w}) \overline{\bar{w}} \\
& -\left(c_{S} \delta\right)^{2}\left|\nabla w+\nabla w^{t}\right|\left(\nabla w+\nabla w^{t}\right) \\
& -v_{F}(\delta) \mathbb{D}(w-\bar{w})-(w-\overline{\bar{w}})-w w \\
= & \widetilde{\mathbb{T}}(w),
\end{aligned}
$$

then $\nabla \cdot \widetilde{\mathbb{T}}(w+W)=\nabla \cdot \widetilde{\mathbb{T}}(w)$ for any constant vector $W$.

Proof. First we consider

$$
\begin{aligned}
\widetilde{\mathbb{T}}(w+W)= & (\overline{w+W})(\overline{w+W})+\overline{(\overline{w+W})(w+W-(\overline{w+W}))} \\
& +\overline{(w+W-(\overline{w+W}))(\overline{w+W})} \\
& -\left(c_{S} \delta\right)^{2}\left|\nabla(w+W)+\nabla(w+W)^{t}\right|\left(\nabla(w+W)+\nabla(w+W)^{t}\right) \\
& -\left(v_{F}(\delta) \mathbb{D}(w+W-(\overline{w+W}))-(w+W-\overline{\overline{w+W}})\right) \\
& -(w+W)(w+W) .
\end{aligned}
$$


Since $W$ is a constant vector, $\overline{\bar{W}}=W, \bar{W}=W, \overline{W w}=W \bar{w}$, and $\overline{w W}=$ $\bar{w} W$. Thus,

$$
\begin{aligned}
\widetilde{\mathbb{T}}(w+W)= & \bar{w} \bar{w}+\overline{\bar{w}(w-\bar{w})+(w-\bar{w}) \bar{w}} \\
& -\left(c_{S} \delta\right)^{2}\left|\nabla w+\nabla w^{t}\right|\left(\nabla w+\nabla w^{t}\right) \\
& -v_{F}(\delta) \mathbb{D}(w-\bar{w})-(w-\overline{\bar{w}}) \\
& -w w+(\bar{w}-w) W+W(\bar{w}-w) \\
& +\overline{W(w-\bar{w})}+\overline{(w-\bar{w}) W}
\end{aligned}
$$

Hence, we have

$$
\begin{aligned}
\nabla \cdot \widetilde{\mathbb{T}}(w+W)= & \nabla \cdot \tilde{T}(w)+\nabla \cdot(\bar{w}-w) W+\nabla \cdot(W(\bar{w}-w)) \\
& +\nabla \cdot((\bar{w}-\overline{\bar{w}}) W)+\nabla \cdot(W(\bar{w}-\overline{\bar{w}})) .
\end{aligned}
$$

Since the averaging preserves incompressibility [14], that is, $\nabla \cdot w=$ $\nabla \cdot \bar{w}=0$, so we have

$$
\nabla \cdot \widetilde{\mathbb{T}}(w+W)=\nabla \cdot \widetilde{\mathbb{T}}(w)
$$

This completes the proof.

\section{Existence of solutions}

In this section, we consider the question of the existence of weak solutions to the following systems. Thus, we seek $(w, q)$ satisfying

$$
\begin{gathered}
w_{t}+\nabla \cdot(\bar{w} \bar{w})+\nabla \cdot(\overline{\bar{w}(w-\bar{w})+(w-\bar{w}) \bar{w}})-\nabla q-\operatorname{Re}^{-1} \Delta w \\
-A(\delta) w=\bar{f}, \quad \nabla \cdot w=0, \text { in } \Omega \times(0, T] \\
w(x, 0)=g_{\delta} * u_{0}(x) \quad \text { in } \Omega, \\
w\left(x_{j}+L, t\right)=w\left(x_{j}, t\right), \\
\int_{\Omega} \bar{u}_{0} d x=0, \quad \int_{\Omega} \bar{f} d x=0, \quad \int_{\Omega} \bar{q} d x=0 .
\end{gathered}
$$

We will begin by giving the definition of weak solution. Let $D(\Omega)=$ $\left\{\psi \in C_{0}^{\infty}(\Omega): \nabla \cdot \psi=0\right.$ in $\left.\Omega\right\}$, let $H(\Omega)$ be the completion of $D(\Omega)$ in $L^{2}(\Omega)$, let $H^{1}(\Omega)$ be the completion of $D(\Omega)$ in $W^{1,2}(\Omega)$, and let $\psi \in$ $D(\Omega)$.

Definition 2.1. Let $u_{0} \in H(\Omega)$ and $f \in L^{2}\left(\Omega_{T}\right)$. A measurable function $w$ : $\Omega_{T} \rightarrow \mathbb{R}^{n}$ is a weak solution of the problem (2.1) and (2.2) in $\Omega_{T}$ if 
434 Existence of weak solutions for a scale similarity model

(a) $w \in V_{T}=L^{2}\left(0, T ; H^{1}\right) \cap L^{\infty}(0, T ; H)$;

(b) $w$ verifies

$$
\begin{aligned}
\int_{0}^{t}[ & -\operatorname{Re}^{-1}(\nabla w, \nabla \psi)+(\bar{w} \bar{w}, \nabla \psi)+(\bar{w}(w-\bar{w})+(w-\bar{w}) \bar{w}, \nabla \bar{\psi}) \\
- & \left.v_{F}(\delta)(\mathbb{D}(w-\bar{w}), \mathbb{D}(\psi-\bar{\psi}))\right] d s \\
& =-\int_{0}^{t}(\bar{f}, \psi) d s+(w(t), \psi)-\left(w_{0}, \psi\right),
\end{aligned}
$$

where, for $T \in(0, \infty), \Omega_{T}=\Omega \times[0, T]$.

Before we prove the existence of weak solutions of (2.1), (2.2), and (2.3), we give the following lemma which is proved in [8]. Here, we will give this proof briefly.

LEMMA 2.2. Let $b(u, v, w)$ denote the (nonstandard) trilinear form

$$
b(u, v, w):=\int_{\Omega} \bar{u} \bar{v}: \nabla w+[\bar{u}(v-\bar{v})+(u-\bar{u}) \bar{v}]: \nabla \bar{w} d x
$$

Suppose that the averaging used in $L^{2}(\Omega)$ is selfadjoint and commutes with differentiation, $w \in L^{2}(\Omega)$ and $\nabla w \in L^{2}(\Omega)$ are periodic with zero mean. Then

$$
I=\int_{\Omega} \nabla \cdot[\bar{w} \bar{w}+\overline{\bar{w}}(w-\bar{w})+(w-\bar{w}) \bar{w}] \cdot w d x=0
$$

Proof. Integration by parts and using the properties of the averaging operator yield

$$
\begin{aligned}
I & =\int_{\Omega}[\bar{w} \bar{w}+\overline{\bar{w}}(w-\bar{w})+(w-\bar{w}) \bar{w}]: \nabla w d x \\
& =\int_{\Omega}[\bar{w} \bar{w}: \nabla w+\bar{w} w: \nabla \bar{w}-\bar{w} \bar{w}: \nabla \bar{w}+w \bar{w}: \nabla \bar{w}-\bar{w} \bar{w}: \nabla \bar{w}] d x
\end{aligned}
$$

An easy index calculation shows that

$$
\int_{\Omega} u v: \nabla w d x=\int_{\Omega} u \cdot(\nabla w) v d x
$$


which is the more familiar trilinear form. Making this change gives

$$
I=\int_{\Omega}[\bar{w} \cdot(\nabla w) \bar{w}+\bar{w} \cdot(\nabla \bar{w}) w+w \cdot(\nabla \bar{w}) \bar{w}-2 \bar{w} \cdot(\nabla \bar{w}) \bar{w}] d x
$$

Since $\nabla \cdot w=0$, the third term vanishes. By the assumption on the averaging process, $\nabla \cdot \bar{w}=0$, the last term vanishes. We use the usual skewsymmetry property to obtain

$$
\int_{\Omega} \bar{w} \cdot(\nabla w) \bar{w}+\bar{w} \cdot(\nabla \bar{w}) w=0
$$

Thus $I=0$.

THEOREM 2.3. Let $T>0$ and let $\Omega$ be any domain in $\mathbb{R}^{d}$. Then, for any given $u_{0} \in L^{2}(\Omega)$ and $f \in L^{2}\left(\Omega_{T}\right)$, there exists at least one weak solution to (2.1), (2.2), and (2.3) in $\Omega_{T}$.

Proof. We will use the Faedo-Galerkin method following the presentation of Galdi in the Navier-Stokes case [3]. Let $D(\Omega)=:\left\{\psi \in C_{0}^{\infty}: \nabla \cdot \psi=\right.$ 0 in $\Omega$, let $H(\Omega)$ be the completion of $D(\Omega)$ in $L^{2}(\Omega)$, let $H^{1}(\Omega)$ be the completion of $D(\Omega)$ in $W^{1,2}(\Omega)$, and let $\left\{\psi_{r}\right\} \subset D(\Omega)$ be the orthonormal basis of $H(\Omega)$. We will look for approximating solutions $v^{k}$ of problem (2.1), (2.2), and (2.3), which have the form

$$
v^{k}(x, t)=\sum_{r=1}^{k} c_{k r}(t) \psi_{r}(x), \quad k \in \mathbb{N}
$$

In (2.1), we set $w=v^{k}$; multiply by $\psi_{r}$ and integrate over $\Omega$ to obtain

$$
\begin{aligned}
\frac{d}{d t}\left(v^{k}, \psi_{r}\right)-\left(\bar{v}^{k} \bar{v}^{k}, \nabla \psi_{r}\right)+\operatorname{Re}^{-1}\left(\nabla v^{k}, \nabla \psi_{r}\right) \\
\quad+v_{F}(\delta)\left(\mathbb{D}\left(v^{k}-\bar{v}^{k}\right), \mathbb{D}\left(\psi_{r}-\bar{\psi}_{r}\right)\right) \\
\quad-\left(\bar{v}^{k}\left(v^{k}-\bar{v}^{k}\right)+\left(v^{k}-\bar{v}^{k}\right) \bar{v}^{k}, \nabla \bar{\psi}_{r}\right) \\
=\left(\bar{f}, \psi_{r}\right) .
\end{aligned}
$$

Note that since $\nabla \cdot u=0$, it follows that $\Delta u=2 \nabla \cdot \mathbb{D}(u)$. The symmetry of deformation tensor yields

$$
\frac{1}{2}(\nabla u, \nabla v)=(\mathbb{D}(u), \mathbb{D}(v))
$$


436 Existence of weak solutions for a scale similarity model

Thus, we obtain the following equality:

$$
\begin{aligned}
\frac{d}{d t}\left(v^{k}, \psi_{r}\right)-\left(\bar{v}^{k} \bar{v}^{k}, \nabla \psi_{r}\right)+\operatorname{Re}^{-1}\left(\nabla v^{k}, \nabla \psi_{r}\right) \\
\quad+\frac{v_{F}(\delta)}{2}\left(\nabla\left(v^{k}-\bar{v}^{k}\right), \nabla\left(\psi_{r}-\bar{\psi}_{r}\right)\right) \\
\quad-\left(\bar{v}^{k}\left(v^{k}-\bar{v}^{k}\right)+\left(v^{k}-\bar{v}^{k}\right) \bar{v}^{k}, \nabla \bar{\psi}_{r}\right) \\
=\left(\bar{f}, \psi_{r}\right) .
\end{aligned}
$$

If we write (2.11) in (2.14), this represents a system of ordinary differential equations of the form

$$
\begin{gathered}
\frac{d}{d t} c_{k r}(t)-\sum_{i, j=1}^{k} c_{k i} c_{k j}\left(\left(g_{\delta} * \psi_{i}\right)\left(g_{\delta} * \psi_{j}\right), \nabla \psi_{r}\right)+\operatorname{Re}^{-1} \sum_{i=1}^{k} c_{k i}\left(\nabla \psi_{i}, \nabla \psi_{r}\right) \\
+\frac{\mathcal{v}_{F}(\delta)}{2} \sum_{j=1}^{k} c_{k j}\left(\nabla\left(\psi_{j}-g_{\delta} * \psi_{j}\right), \nabla\left(\psi_{r}-g_{\delta} * \psi_{r}\right)\right) \\
-\sum_{i=1}^{k} c_{k i} c_{k j}\left[\left(g_{\delta} * \psi_{i}\right)\left(\psi_{j}-g_{\delta} * \psi_{j}\right)\right. \\
\left.\quad+\left(\psi_{j}-g_{\delta} * \psi_{j}\right)\left(g_{\delta} * \psi_{i}\right), \nabla\left(g_{\delta} * \psi_{r}\right)\right] \\
=\left(\bar{f}, \psi_{r}\right)=\bar{f}_{r}, \quad r=1, \ldots, k,
\end{gathered}
$$

with the initial condition

$$
c_{k r}(0)=c_{0 r}=\left(v_{0}, \psi_{r}\right)
$$

Since $\bar{f}_{r} \in L^{2}(0, T)$ for all $r=1, \ldots, k$, from the elementary theory of ordinary differential equations, we know that the problem which is given by (2.15) and (2.16) admits a unique solution $c_{k r} \in W^{1,2}\left(0, T_{k}\right)$, where $T_{k} \leq T$.

Multiplying (2.15) by $c_{k r}$ and summing over $r$ from 1 to $k$, we get

$$
\begin{aligned}
\frac{1}{2} \frac{d}{d t} \| & v_{t}^{k}\left\|_{2}^{2}-\left(\bar{v}^{k} \bar{v}^{k}, \nabla v^{k}\right)+\frac{\mathcal{v}_{F}(\delta)}{2}\right\| \nabla\left(v^{k}-\bar{v}^{k}\right) \|_{2}^{2} \\
& -\left(\bar{v}^{k}\left(v^{k}-\bar{v}^{k}\right)+\left(v^{k}-\bar{v}^{k}\right) \bar{v}^{k}, \nabla \bar{v}^{k}\right)+\operatorname{Re}^{-1}\left\|\nabla v^{k}\right\|_{2}^{2} \\
& =\left(\bar{f}, v^{k}\right) .
\end{aligned}
$$


We integrate this equality to obtain

$$
\begin{aligned}
\left\|v^{k}\right\|_{2}^{2} & +2 \operatorname{Re}^{-1} \int_{0}^{t}\left\|\nabla v^{k}\right\|_{2}^{2} d s-2 \int_{0}^{t}\left(\bar{v}^{k} \bar{v}^{k}, \nabla v^{k}\right) d s \\
& +v_{F}(\delta) \int_{0}^{t}\left\|\nabla\left(v^{k}-\bar{v}^{k}\right)\right\|_{2}^{2} d s \\
& -2 \int_{0}^{t}\left(\bar{v}^{k}\left(v^{k}-\bar{v}^{k}\right)+\left(v^{k}-\bar{v}^{k}\right) \bar{v}^{k}, \nabla \bar{v}^{k}\right) d s \\
= & 2 \int_{0}^{t}\left(\bar{f}, v^{k}\right) d s+\left\|v_{0 k}\right\|_{2}^{2}
\end{aligned}
$$

with $v_{0 k}=v_{k}(0)$. We consider the third and last terms in the left-hand side of (2.18). We write these two terms in nonstandard trilinear form:

$$
\begin{gathered}
b\left(v^{k}, v^{k}, v^{k}\right)=-2 \int_{\Omega}\left[\bar{v}^{k} \cdot \nabla v^{k} \bar{v}^{k}+\bar{v}^{k} \cdot \nabla \bar{v}^{k} v^{k}-\bar{v}^{k} \cdot \nabla \bar{v}^{k} \bar{v}^{k}\right. \\
\left.+v^{k} \cdot \nabla \bar{v}^{k} \bar{v}^{k}-\bar{v}^{k} \cdot \nabla \bar{v}^{k} \bar{v}^{k}\right] d x .
\end{gathered}
$$

From Lemma 2.2, $I=b\left(v^{k}, v^{k}, v^{k}\right)=0$. In the last equality, we use $I=0$, Schwarz inequality, and Poincaré-Friedrichs inequality, and since $\left\|v_{0 k}\right\| \leq$ $\left\|v_{0}\right\|$, we obtain

$$
\begin{aligned}
\left\|v_{k}\right\|_{2}^{2} & +\operatorname{Re}^{-1} \int_{0}^{t}\left\|\nabla v^{k}\right\|_{2}^{2} d s+v_{F}(\delta) \int_{0}^{t}\left\|\nabla\left(v^{k}-\bar{v}^{k}\right)\right\|_{2}^{2} d s \\
\leq & C \operatorname{Re} \int_{0}^{t}\|\bar{f}\|_{2}^{2} d s+\left\|v_{0}\right\|_{2^{\prime}}^{2}
\end{aligned}
$$

where $C$ is a constant. Then we easily deduce the following bound:

$$
\left\|v_{k}\right\|_{2}^{2}+\operatorname{Re}^{-1} \int_{0}^{t}\left\|\nabla v_{k}^{2}\right\|_{2}^{2} d s \leq M \quad \forall t \in[0, T],
$$

with $M$ independent of $t$ and $k$. We will now investigate the properties of convergence of the sequence $\left\{v_{k}\right\}$ when $k \rightarrow \infty$. To this end we begin to show that, for any fixed $r \in \mathbb{N}$, the sequence of functions

$$
G_{k}^{r}(t) \equiv\left(v_{k}(x, t), \psi_{r}\right)
$$

is uniformly bounded and uniformly continuous in $t \in[0, T]$. The uniform boundness follows at once from (2.21). To show the uniform continuity, integrating (2.14) with respect to $t$ from $s$ to $t$ and using Schwarz 
438 Existence of weak solutions for a scale similarity model

inequality, we obtain

$$
\begin{aligned}
\mid G_{k}^{r}(t) & -G_{k}^{r}(s) \mid \\
= & \left|\left(v^{k}(x, t)-v^{k}(x, s), \psi_{r}\right)\right| \\
\leq & \int_{s}^{t}\left|b\left(v^{k}, v^{k}, \psi_{r}\right)\right| d \tau+\frac{v_{F}(\delta)}{2} \int_{s}^{t}\left\|\nabla\left(v^{k}-\bar{v}^{k}\right)\right\|\left\|\nabla\left(\psi_{r}-\bar{\psi}_{r}\right)\right\| d \tau \\
& \quad+\operatorname{Re}^{-1} \int_{s}^{t}\left\|\nabla v^{k}\right\|\left\|\nabla \psi_{r}\right\| d \tau+\int_{s}^{t}\|\bar{f}\|\left\|\psi_{r}\right\| d \tau .
\end{aligned}
$$

On the other hand, an easy index calculation shows that

$$
\int_{\Omega} u v: \nabla w d x=\int_{\Omega} u \cdot(\nabla w) v d x
$$

which is a more familiar trilinear form. Making this change in the formula

$$
b\left(v^{k}, v^{k}, \psi_{r}\right):=\int_{\Omega}\left(\bar{v}^{k} \bar{v}^{k}: \nabla \psi_{r}+\left(\bar{v}^{k}\left(v^{k}-\bar{v}^{k}\right)+\left(v^{k}-\bar{v}^{k}\right) \bar{v}^{k}\right): \nabla \bar{\psi}_{r}\right) d x
$$

gives

$$
b\left(v^{k}, v^{k}, \psi_{r}\right)=\int_{\Omega} \bar{v}^{k} \cdot \nabla \psi_{r} \bar{v}^{k}+\bar{v}^{k} \cdot \nabla \bar{\psi}_{r} v^{k}+v^{k} \cdot \nabla \bar{\psi}_{r} \bar{v}^{k}-2 \bar{v}^{k} \cdot \nabla \bar{\psi}_{r} \bar{v}^{k} .
$$

By the usual skew-symmetry property of this trilinear form, we obtain

$$
b\left(v^{k}, v^{k}, \psi_{r}\right)=\int_{\Omega}-\bar{v}^{k} \cdot \nabla \bar{v}^{k} \psi_{r}-\bar{v}^{k} \cdot \nabla v^{k} \bar{\psi}_{r}-v^{k} \cdot \nabla \bar{v}^{k} \bar{\psi}_{r}+2 \bar{v}^{k} \cdot \nabla \bar{v}^{k} \bar{\psi}_{r} .
$$

Using Cauchy-Schwarz inequality and Young inequality for convolutions, we get

$$
\begin{aligned}
& \int_{s}^{t}\left|b\left(v^{k}, v^{k}, \psi_{r}\right)\right| \\
& \leq s_{1} \max _{t}\left\|v^{k}(x, t)\right\| \sqrt{t-s}\left(\int_{s}^{t}\left\|\nabla v^{k}\right\|^{2}\right)^{1 / 2} \\
& \quad+s_{2} \max _{t}\left\|v^{k}(x, t)\right\| \sqrt{t-s}\left(\int_{s}^{t}\left\|\nabla v^{k}\right\|^{2}\right)^{1 / 2}
\end{aligned}
$$


where $s_{1}=\max _{x \in \Omega}\left|\psi_{r}(x)\right|$ and $s_{2}=4 \max _{x \in \Omega}\left|\bar{\psi}_{r}(x)\right|$. Now we use this inequality and triangle inequality in (2.23) to obtain

$$
\begin{aligned}
\mid G_{k}^{r}(t) & -G_{k}^{r}(s) \mid \\
\leq & \max _{t}\left\|v_{k}(x, t)\right\| \sqrt{t-s}\left\{s_{1}\left(\int_{s}^{t}\left\|\nabla v_{k}\right\|^{2}\right)^{1 / 2}+s_{2}\left(\int_{s}^{t}\left\|\nabla v^{k}\right\|^{2}\right)^{1 / 2}\right\} \\
& +\frac{v_{F}(\delta)}{2} s_{3} \sqrt{t-s}\left(\int_{s}^{t}\left\|\nabla v^{k}\right\|^{2}\right)^{1 / 2} \\
& +\operatorname{Re}^{-1}\left\|\nabla \psi_{r}\right\| \sqrt{t-s}\left(\int_{s}^{t}\left\|\nabla v^{k}\right\|^{2}\right)^{1 / 2} \\
& +\max _{x \in \Omega}\left\|\psi_{r}\right\| \sqrt{t-s}\left(\int_{s}^{t}\|f\|^{2}\right)^{1 / 2},
\end{aligned}
$$

where $s_{3}=2\left\|\nabla \psi_{r}\right\|$. Because of (2.21), the right-hand side of (2.29) converges to zero uniformly as $t \rightarrow s$. The sequence of functions $G_{k}^{r}(t)$ is an equicontinuity. By the Ascoli-Arzela theorem, from the sequence $\left\{G_{k}^{r}(t)\right\}_{k \in \mathbb{N}}$, we may then select a subsequence which we continue to denote by $\left\{G_{k}^{r}(t)\right\}_{k \in \mathbb{N}}$ uniformly converging to a continuous function $G^{r}(t)$. The selected sequence $\left\{G_{k}^{r}(t)\right\}_{k \in \mathbb{N}}$ may depend on $r$. However, using Cantor diagonalization method, we end up with a sequence again denoted by $\left\{G_{k}^{r}(t)\right\}_{k \in \mathbb{N}}$ converging to $G^{r}$ for all $r \in \mathbb{N}$ uniformly in $t \in[0, T]$. This information, together with (2.21) and the weak compactness of the space $H$, allows us to infer the existence of $v(t) \in H(\Omega)$ such that

$$
\lim _{k \rightarrow \infty}\left(v_{k}(t)-v(t), \psi_{r}\right)=0, \quad \text { uniformly in } t \in[0, T], \forall r \in \mathbb{N},
$$

where $v_{k}(t)$ converges weakly in $L^{2}$ to $v(t)$, uniformly in $t \in[0, T]$, that is,

$$
\lim _{k \rightarrow \infty}\left(v_{k}(t)-v(t), u\right)=0, \quad \text { uniformly in } t \in[0, T], \forall u \in L^{2}(\Omega) .
$$

In view of (2.21), $v \in L^{\infty}(0, T ; H(\Omega))$. Again, from (2.21), by the weak compactness of the space $L^{2}\left(\Omega_{T}\right)$,

$$
\lim _{k \rightarrow \infty} \int_{0}^{t}\left(\partial_{m}\left(v_{k}-v\right), w\right) d s=0 \quad \forall w \in L^{2}\left(\Omega_{T}\right), m=1, \ldots, n,
$$

with $\partial_{m}=\partial / \partial x_{m}$ and $v \in L^{2}\left(0, T ; H^{1}(\Omega)\right)$ [3]. It is shown that (2.30) implies the strong convergence of $\left\{v_{k}\right\}$ to $v$ in $L^{2}(w \times[0, T])$ for all $w \subset \Omega$, 
that is,

$$
\lim _{k \rightarrow \infty} \int_{0}^{T}\left\|v_{k}(t)-v(t)\right\|_{2, Q}^{2} d t=0
$$

in [3], where $Q$ is a cube in $\mathbb{R}^{n}$. Now, with the help of (2.31), (2.32), and (2.33), we show that $v$ is a weak solution to (2.1) and (2.2). Since we have already proved that $v \in V_{T}$, it remains to show that $v$ satisfies (2.3). Integrating (2.14) from 0 to $t<T$, we find

$$
\begin{aligned}
-\operatorname{Re}^{-1} & \int_{0}^{t}\left(\nabla v^{k}, \nabla \psi_{r}\right) d s+\int_{0}^{t}\left(\bar{v}^{k}\left(v^{k}-\bar{v}^{k}\right)+\left(v^{k}-\bar{v}^{k}\right) \bar{v}^{k}, \nabla \bar{\psi}_{r}\right) d s \\
& +\int_{0}^{t}\left(\bar{v}^{k} \bar{v}^{k}, \nabla \psi_{r}\right)-\frac{v_{F}(\delta)}{2} \int_{0}^{t}\left(\nabla\left(v^{k}-\bar{v}^{k}\right), \nabla\left(\psi_{r}-\bar{\psi}_{r}\right)\right) d s \\
= & -\int_{0}^{t}\left(\bar{f}, \psi_{r}\right) d s+\left(v^{k}(t), \psi_{r}\right)-\left(v_{o}, \psi_{r}\right) .
\end{aligned}
$$

Now we consider the second and third terms of the left-hand side of (2.34) by the usual skew-symmetry property, writing

$$
\begin{aligned}
& b\left(v^{k}, v^{k}, \psi_{r}\right) \\
& \quad=\int_{0}^{t} \int_{\Omega}\left[-\bar{v}^{k} \cdot \nabla \bar{v}^{k} \psi_{r}-\bar{v}^{k} \cdot \nabla v^{k} \bar{\psi}_{r}-v^{k} \cdot \nabla \bar{v}^{k} \bar{\psi}_{r}+2 \bar{v}^{k} \cdot \nabla \bar{v}^{k} \bar{\psi}_{r}\right] d x .
\end{aligned}
$$

From (2.31) and (2.32), we get

$$
\begin{gathered}
\lim _{k \rightarrow \infty}\left(v^{k}(t)-v(t), \psi_{r}\right)=0 \\
\lim _{k \rightarrow \infty} \int_{0}^{t}\left(\nabla v^{k}(s)-\nabla v(s), \nabla \psi_{r}\right) d s=0 .
\end{gathered}
$$

Furthermore, let $Q$ be a cube containing the support of $\psi_{r}$, then we have

$$
\begin{aligned}
& \left|\int_{0}^{t}\left[\left(\bar{v}^{k} \cdot \nabla \bar{v}^{k}, \psi_{r}\right)-\left(\bar{v} \cdot \nabla \bar{v}, \psi_{r}\right)\right] d s\right| \\
& \quad \leq\left|\int_{0}^{t}\left(\left(\bar{v}^{k}-\bar{v}\right) \cdot \nabla \bar{v}^{k}, \psi_{r}\right)_{Q} d s\right|+\left|\int_{0}^{t}\left(\bar{v} \cdot \nabla\left(\bar{v}^{k}-\bar{v}\right), \psi_{r}\right)_{Q} d s\right| .
\end{aligned}
$$

We consider the first term of the right-hand side of (2.37), and using Cauchy-Schwarz inequality, we obtain

$$
\left|\int_{0}^{t}\left(\left(\bar{v}^{k}-\bar{v}\right) \cdot \nabla \bar{v}^{k}, \psi_{r}\right)_{Q}\right| \leq \int_{0}^{t}\left\|\bar{v}^{k}-\bar{v}\right\|\left\|\nabla \bar{v}^{k}\right\| \max _{x \in Q}\left|\psi_{r}(x)\right| .
$$


Setting $s_{1}: \max _{x \in Q}\left|\psi_{r}(x)\right|$ and using (2.21) and Young inequality for convolution, we have

$$
\left|\int_{0}^{t}\left(\left(\bar{v}^{k}-\bar{v}\right) \cdot \nabla \bar{v}^{k}, \psi_{r}\right)_{Q}\right| \leq C s_{1} M^{1 / 2}\left(\int_{0}^{t}\left\|v^{k}-v\right\|_{2, Q}^{2}\right)^{1 / 2},
$$

where $C$ is a constant. Thus, using (2.33), we get

$$
\lim _{k \rightarrow \infty}\left|\int_{0}^{t}\left(\left(\bar{v}^{k}-\bar{v}\right) \cdot \nabla \bar{v}^{k}, \psi_{r}\right)_{Q} d s\right|=0
$$

We also have

$$
\begin{aligned}
\left|\int_{0}^{t}\left(\bar{v} \cdot \nabla\left(\bar{v}^{k}-\bar{v}\right), \psi_{r}\right)_{Q} d s\right| & \leq \sum_{m=1}^{n}\left|\int_{0}^{t}\left(\partial_{m}\left(\bar{v}^{k}-\bar{v}\right), \bar{v}_{m} \psi_{r}\right)_{Q} d s\right| \\
& \leq \sum_{m=1}^{n}\left|\int_{0}^{t}\left(\partial_{m}\left(v^{k}-v\right), g_{\delta} *\left(\left(g_{\delta} * v_{m}\right) \psi_{r}\right)\right)_{Q} d s\right|,
\end{aligned}
$$

and since $g_{\delta} *\left(\left(g_{\delta} * v_{i}\right) \psi_{r}\right) \in L^{2}\left(\Omega_{T}\right),(2.32)$ implies that

$$
\lim _{k \rightarrow \infty}\left|\int_{0}^{t}\left(\bar{v} \cdot \nabla\left(\bar{v}^{k}-\bar{v}\right), \psi_{r}\right)_{Q} d s\right|=0
$$

Relations (2.40) and (2.42) yield

$$
\lim _{k \rightarrow \infty}\left|\int_{0}^{t}\left(\left(\bar{v}^{k} \cdot \nabla \bar{v}^{k}, \psi_{r}\right)-\left(\bar{v} \cdot \nabla \bar{v}, \psi_{r}\right)\right) d s\right|=0
$$

Now we consider the second term of $b\left(v^{k}, v^{k}, \psi_{r}\right)$ which is given by (2.35). Again let $Q$ be a cube containing the support of $\psi_{r}$, then we have

$$
\begin{aligned}
& \left|\int_{0}^{t}\left(\left(\bar{v}^{k} \cdot \nabla v^{k}, \bar{\psi}_{r}\right)-\left(\bar{v} \cdot \nabla v, \bar{\psi}_{r}\right)\right) d s\right| \\
& \quad \leq\left|\int_{0}^{t}\left(\left(\bar{v}^{k}-\bar{v}\right) \cdot \nabla v^{k}, \bar{\psi}_{r}\right)_{Q} d s\right|+\left|\int_{0}^{t}\left(\bar{v} \cdot \nabla\left(v^{k}-v\right), \bar{\psi}_{r}\right)_{Q} d s\right| .
\end{aligned}
$$


442 Existence of weak solutions for a scale similarity model

We use Cauchy-Schwarz inequality and the first term of the right-hand side of (2.44) to obtain

$$
\begin{aligned}
& \left|\int_{0}^{t}\left(\left(\bar{v}^{k}-\bar{v}\right) \cdot \nabla v^{k}, \bar{\psi}_{r}\right)_{Q} d s\right| \\
& \quad \leq s_{2}\left(\int_{0}^{t}\left\|\bar{v}^{k}-\bar{v}\right\|_{2, Q}^{2} d s\right)^{1 / 2}\left(\int_{0}^{t}\left\|\nabla v^{k}\right\|_{2, Q}^{2} d s\right)^{1 / 2} .
\end{aligned}
$$

Using (2.21) and Young inequality, we get

$$
\left|\int_{0}^{t}\left(\left(\bar{v}^{k}-\bar{v}\right) \cdot \nabla v^{k}, \bar{\psi}_{r}\right)_{Q} d s\right| \leq C s_{2} M^{1 / 2}\left(\int_{0}^{t}\left\|v^{k}-v\right\|_{2, Q}^{2} d s\right)^{1 / 2}
$$

Thus, using (2.33), we obtain

$$
\lim _{k \rightarrow \infty}\left|\int_{0}^{t}\left(\left(\bar{v}^{k}-\bar{v}\right) \cdot \nabla v^{k}, \bar{\psi}_{r}\right)_{Q} d s\right|=0
$$

Now we consider the second term of the right-hand side of (2.44); we write

$$
\left|\int_{0}^{t}\left(\bar{v}^{k} \cdot \nabla\left(v^{k}-v\right), \bar{\psi}_{r}\right)_{Q}\right| \leq \sum_{m=1}^{n}\left|\int_{0}^{t}\left(\partial_{m}\left(v^{k}-v\right), \bar{v}_{m} \bar{\psi}_{r}\right)_{Q} d s\right|
$$

and since $\bar{v}_{m} \bar{\psi}_{r} \in L^{2}\left(\Omega_{T}\right)$, (2.32) implies

$$
\lim _{k \rightarrow \infty}\left|\int_{0}^{t}\left(\bar{v}^{k} \cdot \nabla\left(v^{k}-v\right), \bar{\psi}_{r}\right)_{Q} d s\right|=0
$$

Relations (2.47) and (2.49) yield

$$
\lim _{k \rightarrow \infty}\left|\int_{0}^{t}\left(\left(\bar{v}^{k} \cdot \nabla v^{k}, \bar{\psi}_{r}\right)-\left(\bar{v} \cdot \nabla v, \bar{\psi}_{r}\right)\right) d s\right|=0
$$

Similarly we consider the third term of $b\left(v^{k}, v^{k}, \psi_{r}\right)$ which is given by (2.35); we write

$$
\begin{aligned}
& \left|\int_{0}^{t}\left[\left(v^{k} \cdot \nabla \bar{v}^{k}, \bar{\psi}_{r}\right)-\left(v \cdot \nabla \bar{v}, \bar{\psi}_{r}\right)\right] d s\right| \\
& \quad \leq\left|\int_{0}^{t}\left(\left(v^{k}-v\right) \cdot \nabla \bar{v}^{k}, \bar{\psi}_{r}\right)_{Q} d s\right|+\left|\int_{0}^{t}\left(v \cdot \nabla\left(\bar{v}^{k}-\bar{v}\right), \bar{\psi}_{r}\right)_{Q}\right| .
\end{aligned}
$$


Again using Cauchy-Schwarz inequality, Young inequality, and (2.21) in the first term of the right-hand side of (2.51), we get

$$
\left|\int_{0}^{t}\left(\left(v^{k}-v\right) \cdot \nabla \bar{v}^{k}, \bar{\psi}_{r}\right)_{Q} d s\right| \leq C s_{2} M^{1 / 2}\left(\int_{0}^{t}\left\|v^{k}-v\right\|_{2, Q}^{2} d s\right)^{1 / 2}
$$

Using (2.33), we get

$$
\lim _{k \rightarrow \infty}\left|\int_{0}^{t}\left(\left(v^{k}-v\right) \cdot \nabla \bar{v}^{k}, \bar{\psi}_{r}\right)_{Q} d s\right|=0 .
$$

Now we consider the second term of the right-hand side of (2.51)

$$
\left|\int_{0}^{t}\left(v \cdot \nabla\left(\bar{v}^{k}-\bar{v}\right), \bar{\psi}_{r}\right)_{Q} d s\right| \leq \sum_{m=1}^{n}\left|\int_{0}^{t}\left(\partial_{m}\left(\bar{v}^{k}-\bar{v}\right), v_{m} \bar{\psi}_{r}\right)_{Q} d s\right| .
$$

We use the properties of convolutions to obtain

$$
\left|\int_{0}^{t}\left(v \cdot \nabla\left(\bar{v}^{k}-\bar{v}\right), \bar{\psi}_{r}\right)_{Q} d s\right| \leq \sum_{m=1}^{n}\left|\int_{0}^{t} \partial_{m}\left(v^{k}-v\right), g_{\delta} *\left(v_{m} \bar{\psi}_{r}\right)_{Q} d s\right| \text {. }
$$

Since $g_{\delta} *\left(v_{m} \bar{\psi}_{r}\right) \in L^{2}\left(\Omega_{T}\right),(2.32)$ implies

$$
\lim _{k \rightarrow \infty}\left|\int_{0}^{t}\left(v \cdot \nabla\left(\bar{v}^{k}-\bar{v}\right), \bar{\psi}_{r}\right)_{Q} d s\right|=0
$$

Relations (2.53) and (2.56) yield

$$
\lim _{k \rightarrow \infty}\left|\int_{0}^{t}\left[\left(v^{k} \cdot \nabla \bar{v}^{k}, \bar{\psi}_{r}\right)-\left(v \cdot \nabla \bar{v}, \bar{\psi}_{r}\right)\right] d s\right|=0 .
$$

Now we consider the last term of $b\left(v^{k}, v^{k}, \psi_{r}\right)$ which is given by (2.35). Again we can write

$$
\begin{aligned}
& \left|\int_{0}^{t}\left[\left(\bar{v}^{k} \cdot \nabla \bar{v}^{k}, \bar{\psi}_{r}\right)-\left(\bar{v} \cdot \nabla \bar{v}, \bar{\psi}_{r}\right)\right] d s\right| \\
& \quad \leq\left|\int_{0}^{t}\left(\left(\bar{v}^{k}-\bar{v}\right) \cdot \nabla \bar{v}^{k}, \bar{\psi}_{r}\right)_{Q} d s\right|+\left|\int_{0}^{t}\left(\bar{v} \cdot \nabla\left(\bar{v}^{k}-\bar{v}\right), \bar{\psi}_{r}\right)_{Q} d s\right| .
\end{aligned}
$$

Similarly, using Cauchy-Schwarz inequality, Young inequality, (2.21), and (2.33) in the first term of the right-hand side of (2.58), we get

$$
\lim _{k \rightarrow \infty}\left|\int_{0}^{t}\left(\left(\bar{v}^{k}-\bar{v}\right) \cdot \nabla \bar{v}^{k}, \bar{\psi}_{r}\right)_{Q} d s\right|=0
$$


444 Existence of weak solutions for a scale similarity model

Besides, we get the following inequality for the second term of (2.58):

$$
\left|\int_{0}^{t}\left(\bar{v} \cdot \nabla\left(\bar{v}^{k}-\bar{v}\right), \bar{\psi}_{r}\right)_{Q} d s\right| \leq \sum_{m=1}^{n}\left|\int_{0}^{t}\left(\partial_{m}\left(\bar{v}^{k}-\bar{v}\right), \bar{v}_{m} \bar{\psi}_{r}\right)_{Q}\right| .
$$

From the properties of convolution, we write

$$
\left|\int_{0}^{t}\left(\bar{v} \cdot \nabla\left(\bar{v}^{k}-\bar{v}\right), \bar{\psi}_{r}\right)_{Q} d s\right| \leq \sum_{m=1}^{n}\left|\int_{0}^{t}\left(\partial_{m}\left(v^{k}-v\right), g_{\delta} *\left(\bar{v}_{m} \bar{\psi}_{r}\right)\right)_{Q}\right| \text {. }
$$

Since $g_{\delta} *\left(\bar{v}_{m} \bar{\psi}_{r}\right) \in L^{2}\left(\Omega_{T}\right)$, and from (2.32), we obtain

$$
\lim _{k \rightarrow \infty}\left|\int_{0}^{t}\left(\bar{v} \cdot \nabla\left(\bar{v}^{k}-\bar{v}\right), \bar{\psi}_{r}\right)_{Q} d s\right|=0
$$

Thus, relations (2.59) and (2.62) yield

$$
\lim _{k \rightarrow \infty}\left|\int_{0}^{t}\left[\left(\bar{v}^{k} \cdot \nabla \bar{v}^{k}, \bar{\psi}_{r}\right)-\left(\bar{v} \cdot \nabla \bar{v}, \bar{\psi}_{r}\right)\right] d s\right|=0 .
$$

Finally, we consider the fourth term of the left-hand side of (2.34). Again let $Q$ be a cube containing the support of $\psi_{r}$, then we have

$$
\begin{aligned}
\left|\int_{0}^{t}\left[\left(\nabla\left(v^{k}-v\right), \nabla\left(\psi_{r}-\bar{\psi}_{r}\right)\right)-\left(\nabla\left(\bar{v}^{k}-\bar{v}\right), \nabla\left(\psi_{r}-\bar{\psi}_{r}\right)\right)\right] d s\right| \\
\leq\left|\int_{0}^{t}\left(\nabla\left(v^{k}-v\right), \nabla\left(\psi_{r}-\bar{\psi}_{r}\right)\right)_{Q} d s\right| \\
\quad+\left|\int_{0}^{t}\left(\nabla\left(\bar{v}^{k}-\bar{v}\right), \nabla\left(\psi_{r}-\bar{\psi}_{r}\right)\right)_{Q} d s\right| .
\end{aligned}
$$

Since $\nabla\left(\psi_{r}-\bar{\psi}_{r}\right) \in L^{2}\left(\Omega_{T}\right)$, and using (2.32), we get

$$
\lim _{k \rightarrow \infty}\left|\int_{0}^{t}\left(\nabla\left(v^{k}-v\right), \nabla\left(\psi_{r}-\bar{\psi}_{r}\right)\right)_{Q}\right|=0 .
$$

Similarly, since $g_{\delta} * \nabla\left(\psi_{r}-\bar{\psi}_{r}\right) \in L^{2}\left(\Omega_{T}\right)$, and using (2.32), it gives

$$
\lim _{k \rightarrow \infty}\left|\int_{0}^{t}\left(\nabla\left(\bar{v}^{k}-\bar{v}\right), \nabla\left(\psi_{r}-\bar{\psi}_{r}\right)\right)_{Q} d s\right|=0
$$

Using (2.65) and (2.66), we get

$$
\lim _{k \rightarrow \infty}\left|\int_{0}^{t}\left(\nabla\left(v^{k}-\bar{v}^{k}\right)-\nabla(v-\bar{v}), \nabla\left(\psi_{r}-\bar{\psi}_{r}\right)\right) d s\right|=0 .
$$


Therefore, taking the limit over $k \rightarrow \infty$ in (2.34) and using (2.36), (2.43), (2.50), (2.57), (2.63), and (2.67), we get

$$
\begin{aligned}
-\operatorname{Re}^{-1} & \int_{0}^{t}\left(\nabla v, \nabla \psi_{r}\right)+\int_{0}^{t}\left(\bar{v}(v-\bar{v})+(v-\bar{v}) \bar{v}, \nabla \bar{\psi}_{r}\right) d s \\
& +\int_{0}^{t}\left(\bar{v} \bar{v}, \nabla \psi_{r}\right) d s-\frac{v_{F}(\delta)}{2} \int_{0}^{t}\left(\nabla(v-\bar{v}), \nabla\left(\psi_{r}-\bar{\psi}_{r}\right)\right) \\
= & -\int_{0}^{t}\left(\bar{f}, \psi_{r}\right) d s+\left(v(t), \psi_{r}\right)-\left(v_{0}, \psi_{r}\right) .
\end{aligned}
$$

However, from [3, Lemma 2.3], we know that every function $\psi \in D(\Omega)$ can be uniformly approximated in $C^{2}(\bar{\Omega})$ by functions of the form

$$
\psi_{N}(x)=\sum_{r=1}^{N} \gamma_{r} \psi_{r}(x), \quad N \in \mathbb{N}, \gamma_{r} \in \mathbb{R} .
$$

So we write (2.68) with $\psi_{N}$ in place of $\psi_{r}$ and we may pass to the limit $N \rightarrow \infty$ in this new relation and use the fact that $v \in L^{2}\left(0, T ; H^{1}\right) \cap L^{\infty}(0, T$; $H$ ) to show that $v$ is a weak solution of (2.1) and (2.2).

\section{Acknowledgments}

I would like to thank Prof. Dr. W. J. Layton for this problem, his valuable comments, and several helpful discussions. The research of M. Kaya was conducted during a visit to the University of Pittsburgh.

\section{References}

[1] J. Bardina, J. Ferziger, and W. Reynolds, Improved subgrid scale models for large eddy simulation, AIAA paper 80-1357, 1980.

[2] Q. Du and M. Gunzburger, Analysis of a Ladyzhenskaya model for incompressible viscous flow, J. Math. Anal. Appl. 155 (1991), no. 1, 21-45.

[3] G. P. Galdi, An introduction to the Navier-Stokes initial-boundary value problem, Fundamental Directions in Mathematical Fluid Mechanics (G. P. Galdi, J. G. Heywood, and Rannacher R., eds.), Adv. Math. Fluid Mech., Birkhäuser Verlag, Basel, 2000, pp. 1-70.

[4] K. Horiuti, Backward scatter of subgrid-scale energy in wall-bounded and free shear turbulence, J. Phys. Soc. Japan 66 (1997), 91-107.

[5] T. Iliescu and W. J. Layton, Approximating the larger eddies in fluid motion. III. The Boussinesq model for turbulent fluctuations, An. Şti. Univ. "Al. I. Cuza" Iaşi Secţ. I a Mat. 44 (1998), no. 2, 245-261.

[6] O. A. Ladyzhenskaya, New equations for the description of the motions of viscous incompressible fluids, and global solvability for their boundary value problems, Trudy Mat. Inst. Steklov. 102 (1967), 85-104. 


\section{Existence of weak solutions for a scale similarity model}

[7] W. J. Layton, A nonlinear, subgrid-scale model for incompressible viscous flow problems, SIAM J. Sci. Comput. 17 (1996), no. 2, 347-357.

[8] Approximating the larger eddies in fluid motion. $V$. Kinetic energy balance of scale similarity models, Math. Comput. Modelling 31 (2000), no. 8-9, 1-7.

[9] A. Leonard, Energy cascade in large-eddy simulations of turbulent fluid flows, Adv. Geophys 18 (1974), 237-248.

[10] S. Liu, C. Meneveau, and J. Katz, On properties of similarity subgrid-scale models as deduced from measurements in a turbulent jet, J. Fluid Mech. 275 (1994), 83-119.

[11] P. Sagaut, Large Eddy Simulation for Incompressible Flows, Springer-Verlag, New York, 1998.

[12] F. Sarghini, U. Piomelli, and E. Balaras, Scale-similar models for large eddy simulations, Phys. Fluids 11 (1999), 1596-1607.

[13] J. Smagorinsky, General circulation experiments with the primitive equations. I. The basic experiment, Mon. Wea. Rev. 91 (1963), 99-164.

[14] C. G. Speziale, Galilean invariance of subgrid-scale stress models in the large-eddy simulation of turbulence, J. Fluid Mech. 156 (1985), 55-62.

Meryem Kaya: Department of Mathematics, Faculty of Arts and Sciences, Gazi University, 06500 Teknikokullar, Ankara, Turkey

E-mail address: meryemk@gazi.edu.tr 


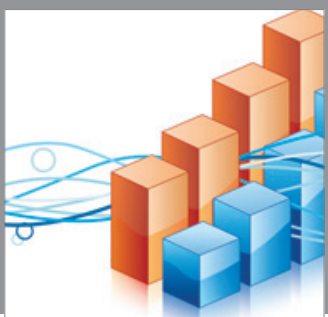

Advances in

Operations Research

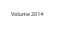

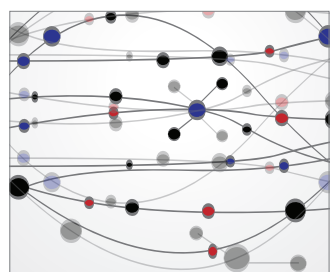

\section{The Scientific} World Journal
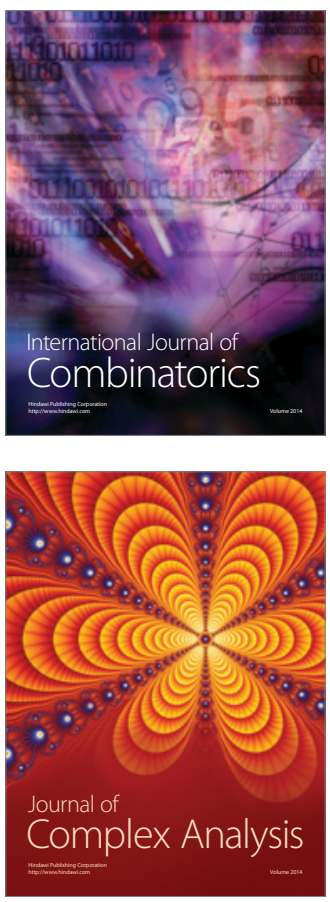

International Journal of

Mathematics and

Mathematical

Sciences
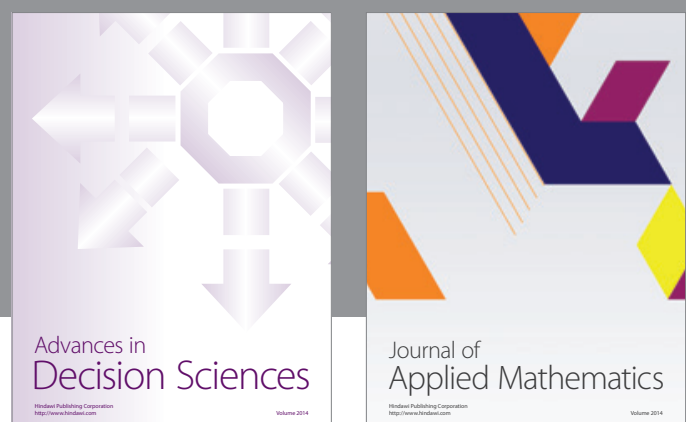

Journal of

Applied Mathematics
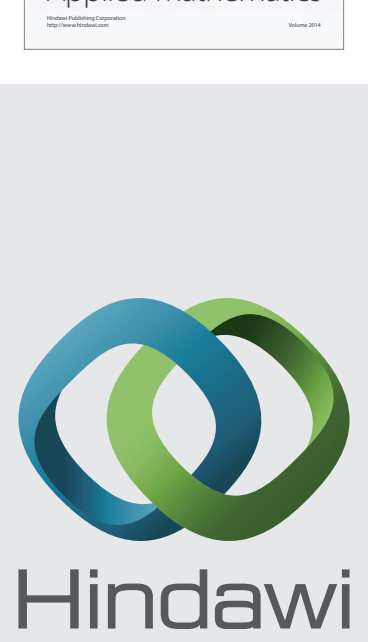

Submit your manuscripts at http://www.hindawi.com
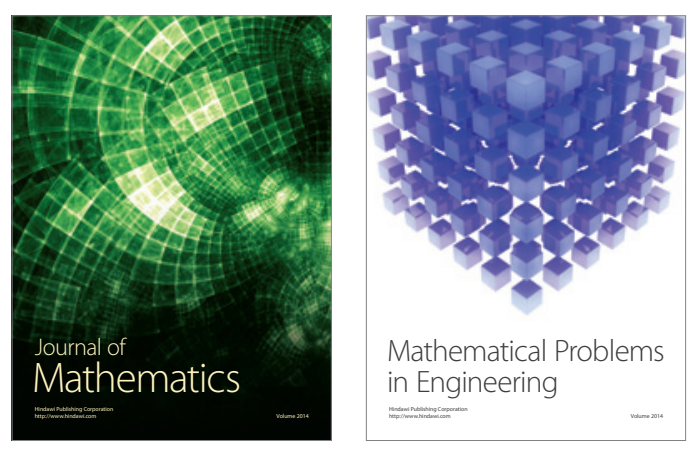

Mathematical Problems in Engineering
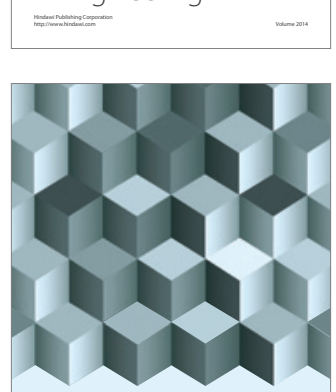

Journal of

Function Spaces
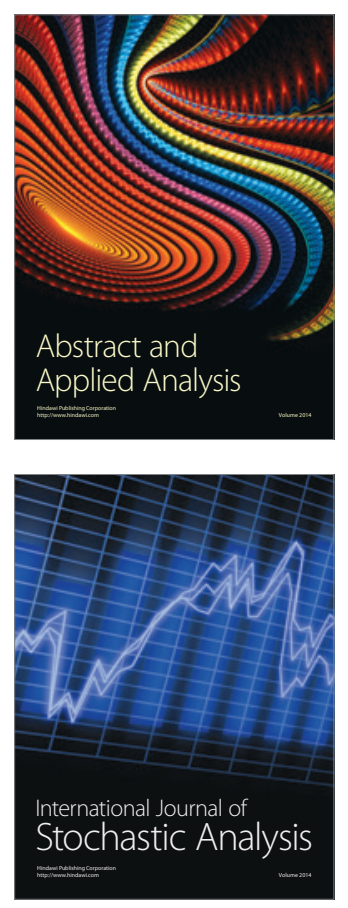

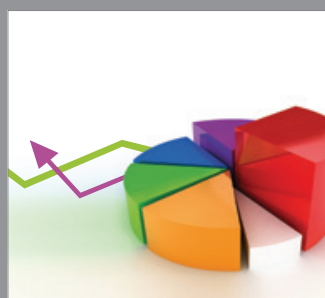

ournal of

Probability and Statistics

Promensencen
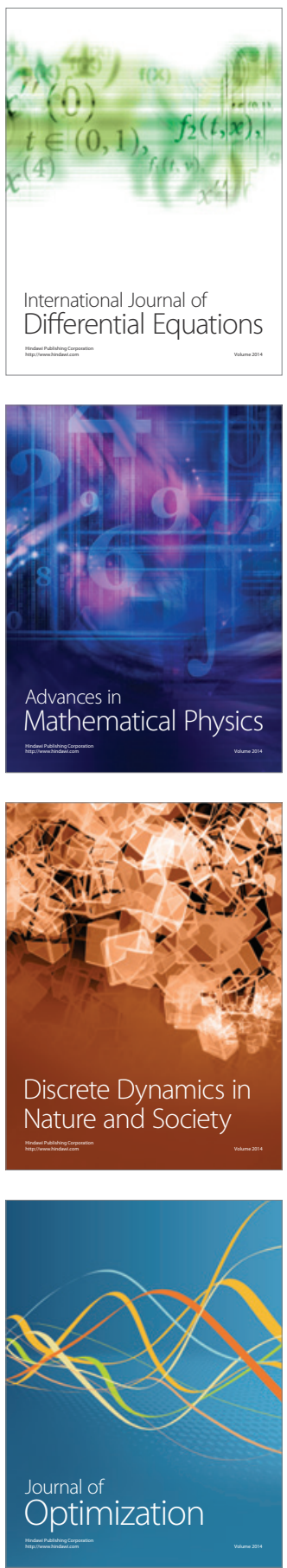